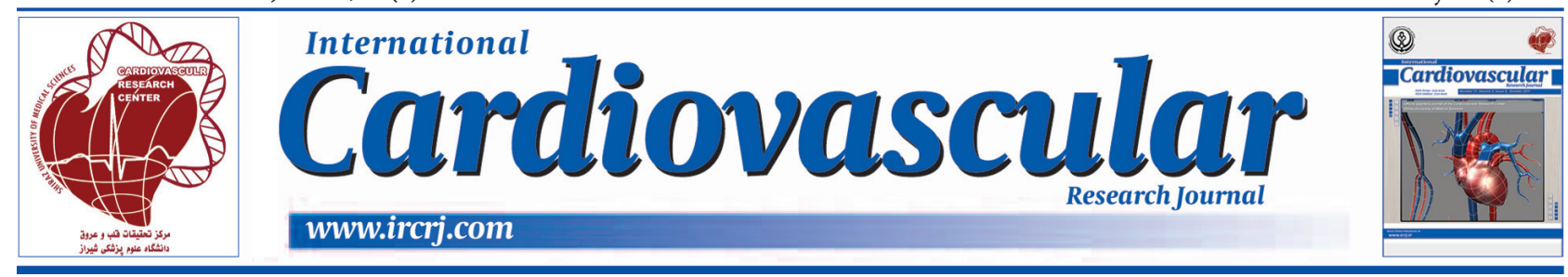

\title{
The Effect of Vitamin D Deficiency Treatment on Post-PCI Coronary Restenosis and Major Adverse Cardiac Events
}

\author{
Zahra Jozanikohan 1,* and Davood Kazemi Saleh²
}

${ }^{1}$ Department of Cardiology, Islamic Azad University, Tehran Medical Sciences Branch, Tehran, IR Iran

${ }^{2}$ Atherosclerosis Research Center, Baqiyatallah University of Medical Sciences, Tehran, IR Iran

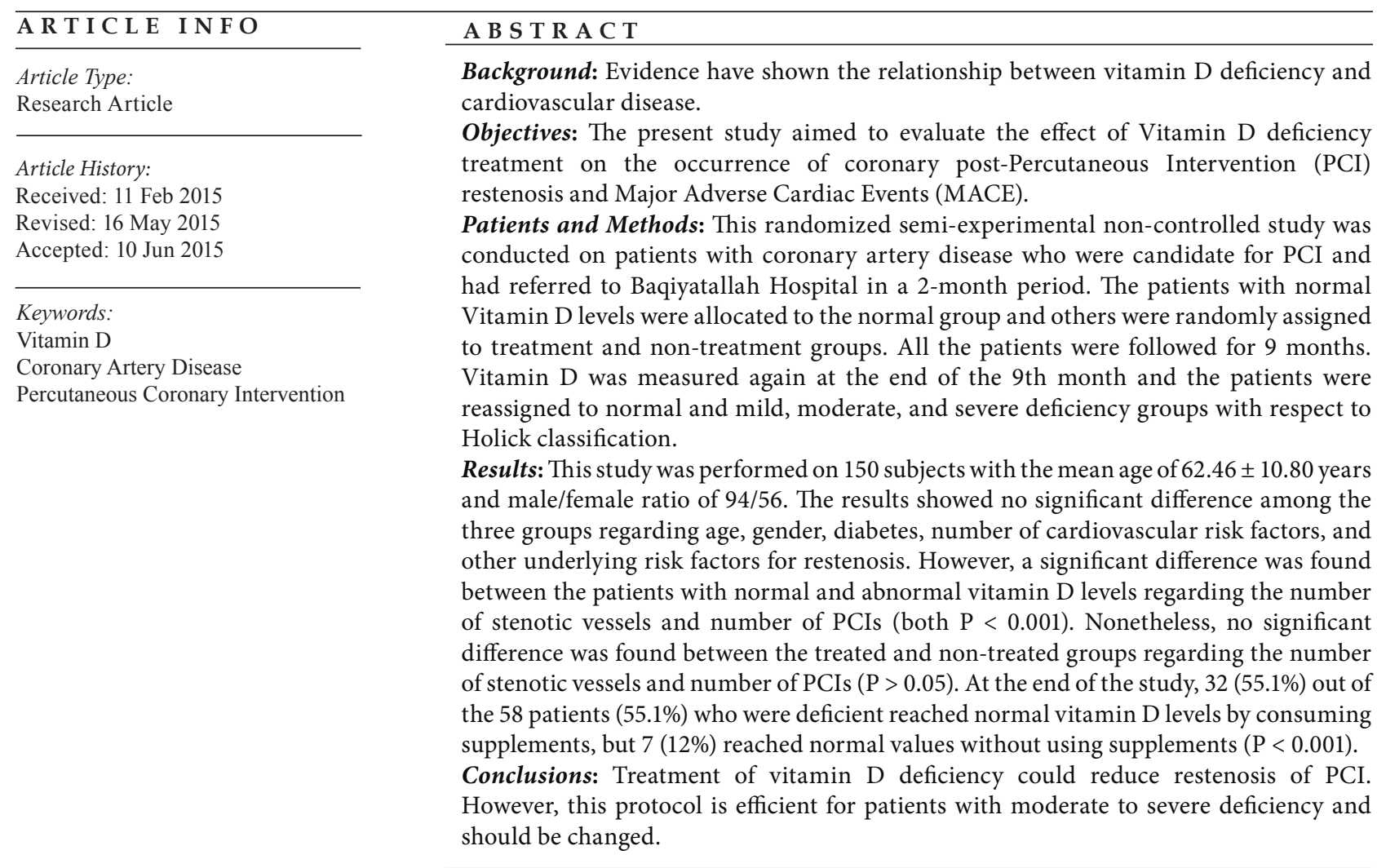

Implication for health policy/practice/research/medical education:

Treatment of vitamin D deficiency could reduce restenosis of percutaneous coronary intervention. However, this protocol is efficient for patients with moderate to severe deficiency and should be changed. Thus, more detailed observations and trials with larger samples sizes are recommended to be conducted on the issue. Treatment of vitamin D deficiency could reduce restenosis of percutaneous coronary intervention. However, this protocol is efficient for patients with moderate to severe deficiency and should be changed. Thus, more detailed observations and trials with larger samples sizes are recommended to be conducted on the issue.

\section{Background}

Vitamin D3 (cholecalciferol) is naturally made

*Corresponding author: Zahra Jozanikohan, Department of Cardiology, Islamic Azad University, Tehran Medical Sciences Branch, Tehran, Iran. Cellphone: +98-9121891388, Fax: +98-2182483250

E-mail: zahrajozani@yahoo.com by the action of ultraviolet-B (UVB) radiation on 7-dehydrocholesterol in the skin followed by a thermal process. Vitamin D3 is converted to 25-hydroxyVitamin D3 $(25(\mathrm{OH}) \mathrm{D})$ in hepatocytes (the circulating form) and then to 1,25-dihydroxyVitamin D3 $(1,25(\mathrm{OH}) 2 \mathrm{D} 3)$ in kidneys (the active form) for further action (1-3). 
Evidence has shown that function of vitamin D is not limited to ions (calcium and magnesium) balance. Studies have also demonstrated that vitamin D plays an important role in preventing cancers, cardiovascular diseases, depression, immune system deficiencies, pregnancy, and neonatal disorders $(4,5)$. Therefore, treating individuals with low serum vitamin D levels, even without any bone malformation, is essential. Several recent observational studies found higher incidence and mortality rates of cardiovascular diseases (coronary heart disease and/ or stroke) among individuals with low serum vitamin D levels $(6,7)$. The treatments include reduction of the risk of metabolic disease through effects on insulin sensitivity, blood pressure, and arterial calcification as well as reducing the risk of infectious diseases and inflammation $(6,7)$. A study on 15000 participants revealed that the prevalence of hypertension, diabetes, and high triglyceride levels was significantly higher in individuals with low vitamin D concentrations (8). Besides, it was shown in a subgroup of patients in the Framingham heart study that those with vitamin D3 levels $<15 \mathrm{ng} / \mathrm{mL}$ had a $60 \%$ greater incidence rate of cardiac events compared to those with higher levels (9). Moreover, women on statin therapy had a higher bone density, presumably mediated through elevation of vitamin D level and improved calcium absorption $(8,9)$.

Patients with low vitamin D levels are not only more prone to cardiovascular disease, but they also have higher recurrence rate of the disease (10). For example, restenosis is a major failure in candidates for Percutaneous Intervention (PCI). There are several recognized risk factors for restenosis, including long stent, small vessel, diabetes, hypertension, dyslipidemia, cigarette smoking, and inherited factors. Retrospective studies have found that several clinical, angiographic, and procedural variables are important predictors of restenosis $(10,11)$. However, the question remains: "what is the role of vitamin D in coronary restenosis".

\section{Objectives}

The present study aims to evaluate the effect of vitamin D deficiency treatment on the occurrence of coronary post-PCI restenosis and Major Adverse Coronary Events (MACE).

\section{Patients and Methods}

\subsection{Subjects}

This randomized, semi-experimental, non-controlled study was extracted from a larger cohort study. The study population consisted of patients with coronary artery disease who were candidate of PCI and had referred to Baqiyatallah Hospital (a referral military-ruled hospital located in Tehran, Iran) from April-May 2013 and were followed for 9 months until February-March 2014. According to the previous studies, the incidence rate of restenosis after PCI was nearly $10 \%$ in the total population. According to the sample size formula, at least 138 individuals were required for the study. Considering $\alpha=0.05$ and power of 0.8 , a 150 -subject sample size was determined for the study. The inclusion criteria of the study were suffering from coronary artery disease and being candidate for PCI with drug-eluted stents. However, the patients with acute or chronic renal failure, acute or chronic liver failure, and mal-absorption disorders were excluded from the study. All the patients signed an informed consent and the research protocol conformed to Declaration of Helsinki. All the information was kept confidential and the results were presented anonymously. The Ethics Committee of Baqiyatallah University of Medical Sciences approved the study and the Research Vice-chancellor of the University granted the study without any third party support (20 January 2013, code 861).

\subsection{Design}

In this single-blind semi-experimental study, blood samples were taken from all the patients and vitamin D3 level was measured by ELISA method using a commercial measuring kit (Euroimmune AG, Germany - inter and intra-assay CI less than 7\%). Since there are no certain classification methods for vitamin D deficiency, the following cut-off points were used for further evaluation: values equal to or more than 30 IU were considered as normal, 29.9 - 20 IU as mild deficiency, 19.9 - 10 IU as moderate deficiency, and $<10$ as severe deficiency. Accordingly, the patients with normal vitamin D levels were allocated to the normal group and others were randomly assigned to two groups using the table of random numbers: 1 . the patients who received treatment (one vitamin D3 pearl of 50,000 IU per week for 10 weeks followed by one pearl per month for maintenance) $(12,13)$ and 2 the patients who did not received the treatment.

All the patients were followed for 9 months and all the cardiac events were recorded (restenosis and MACE). Vitamin D3 level was measured again at the end of the 9th month and the patients were reassigned to the study groups according to the above-mentioned classification. It should be mentioned that all the interventions and follow-ups were observed and recorded by one researcher.

In order to determine restenosis (defined as more than $50 \%$ occlusion in direct observation), all the patients underwent stress echocardiographic study with dobutamine using the method by European Association of Echocardiography (infusion of $5 \mathrm{mcrgr} / \mathrm{kg} / \mathrm{min}$ increasing to $10 \mathrm{mcrgr} / \mathrm{kg}$ / min every 3 minutes and, if needed, up to $40 \mathrm{mcrgr} / \mathrm{kg} /$ min after 12 minutes combined with $1 \mathrm{mg}$ atropine if the heart rate was below our target) (14). Then, the patients with ischemia at PCI territory underwent angiography for checking restenosis.

\subsection{Statistical Evaluation}

The data were expressed as mean \pm Standard Deviation (SD) for quantitative variables (vitamin D and age) and frequency (percentage) for categorical variables (stent type, stent size, lesion type, vitamin D deficiency grouping, risk factors, diabetes mellitus, and number of stenotic coronary arteries). The data were analyzed via the SPSS statistical software (version 20) using chi-square test for categorical variables and t-test and Mann-Whitney-U test for quantitative variables according to their normality and variance equality.

\section{Results}

\subsection{Baseline Characteristics}

This study was performed on 150 subjects with the mean 
age of $62.46 \pm 10.80$ years and male/female ratio of $94 / 56$ $(62 \% / 38 \%)$. The mean number of risk factors was $1.48 \pm$ 1.06 , and 47 subjects $(31.1 \%)$ had diabetes.

In the first vitamin D measurement, 150 patients were included. Among these patients, 34 had normal vitamin D levels and did not receive treatment, while 116 were deficient and were divided into two groups of 58; with treatment and without treatment. According to Table 1, no significant difference was found among the three groups regarding age, sex, frequency of diabetes, number of risk factors, and underlying risk factors of restenosis. However, a significant difference was found between the normal and abnormal vitamin $\mathrm{D}$ patients regarding the number of stenotic vessels and number of PCIs (both $\mathrm{P}<0.001$ ). Nevertheless, no significant difference was found between the treated and non-treated groups in this respect $(\mathrm{P}>0.05)$.

\subsection{Post-Treatment Grouping}

In post-treatment grouping (second vitamin D measurement), 60 subjects had normal vitamin D levels. In fact, 21 participants with normal vitamin D levels remained normal, 7 were deficient and reached normal levels through physiological processes without any supplements, and 32 were deficient and reached normal levels by using supplements. On the other hand, 82 patients had abnormal vitamin D levels. In fact, 13 subjects were normal but ended up with lower (deficient) levels, 47 were deficient and remained deficient without any treatments, and 22 were deficient and remained deficient even after using supplements. It should be noted that 8 patients were lost to follow-up and 142 subjects were analyzed. According to Table 2, no significant difference was found among the three groups regarding the aforementioned risk factors.

At the end of the study, $32(55.1 \%)$ out of the 58 patients (55.1\%) who were deficient reached normal vitamin D levels by consuming supplements, but $7(12 \%)$ reached normal values without using supplements $(\mathrm{P}<0.001)$.

\subsection{Restenosis and MACE}

Based on stress echocardiographic and re-angiographic examination, 7 subjects, one from the final normal group and 6 from the deficient group, had restenosis $(\mathrm{P}<0.001)$. Furthermore, the patients with normal vitamin D levels did not have any restenosis. In addition, one, no, and six cases of restenosis were detected in the treated patients, those with mild deficiency, and those with moderate to severe deficiency, respectively.

At the end of the study, four patients presented with MACE as follows: 1- one 48-year-old female from the unsuccessful treated group with initial and final severe vitamin D deficiency, Myocardial Infarction (MI), and early definite stent thrombosis following procedure on the Right Circumflex Artery (RCA), 2- an 80-year-old female from the non-treated group with initial severe vitamin D deficiency, late possible stent thrombosis, expired before the end of the study (she had 2 risk factors, including diabetes mellitus, revascularization was performed on the right and left circumflex arteries), 3- an 85-year-old man from the treated group without any risk factors, with initial severe vitamin D deficiency, late possible stent thrombosis, expired before the final vitamin D evaluation (PCI on the left circumflex artery), and 4- a 74-year-old woman from the successful treated group, having diabetes mellitus with initial mild vitamin D deficiency, normal final Vitamin D level, restenosis happened after two months, and finally underwent Coronary Artery Bypass Graft (CABG) (figure 1).

\section{Discussion}

Vitamin D supplementation has certain effects on health. Besides, restenosis is the main drawback of PCI. Up to now,

\begin{tabular}{|c|c|c|c|c|c|}
\hline & Total & Normal Vitamin D & Deficient with Treatment & Deficient without Treatment & $P$ value \\
\hline Age & $62.54 \pm 10.80$ & $65.41 \pm 11.06$ & $62.35 \pm 9.50$ & $61.01 \pm 11.45$ & 0.503 \\
\hline $\operatorname{Sex}(M / F)$ & $94 / 56$ & $21 / 13$ & $33 / 18$ & $48 / 17$ & 0.314 \\
\hline Number of risk factors & $1.48 \pm 1.06$ & $1.41 \pm 1.04$ & $1.72 \pm 1.02$ & $1.33 \pm 1.09$ & 0.125 \\
\hline Diabetes & $47(35.1 \%)$ & $12(34.3 \%)$ & $18(36.3 \%)$ & $17(36.1 \%)$ & 0.278 \\
\hline Number of stenotic vessels & $1.9 \pm 0.79$ & $1.79 \pm 0.72$ & $1.94 \pm 0.83$ & $1.92 \pm 0.79$ & 0.813 \\
\hline Number of stented vessels & $1.24 \pm 0.42$ & $1.23 \pm 0.43$ & $1.19 \pm 0.40$ & $1.28 \pm 0.45$ & 0.382 \\
\hline Stents over $25 \mathrm{~mm}$ length & $78(52.5 \%)$ & $15(44.1 \%)$ & $30(51.7 \%)$ & $32(53.1 \%)$ & 0.096 \\
\hline Stents under $2.5 \mathrm{~mm}$ diameter & 9 & 2 & 4 & 3 & - \\
\hline Multiple stents in one vessel & 25 & 8 & 10 & 7 & 0.412 \\
\hline
\end{tabular}

\begin{tabular}{lllll}
\hline \multicolumn{2}{l}{ Table 2. Comparison of the Study Groups Regarding the Risk Factors } & & \\
\hline & $\begin{array}{l}\text { Total Natural } \\
\text { Normal Vitamin D }\end{array}$ & $\begin{array}{l}\text { Treated Normal } \\
\text { Vitamin D }\end{array}$ & $\begin{array}{l}\text { Mild Vitamin } \\
\text { D Deficiency }\end{array}$ & $\begin{array}{l}\text { Moderate to Severe } \\
\text { Vitamin D Deficiency }\end{array}$ \\
\hline Age & $67.47 \pm 10.54$ & $62.51 \pm 10.30$ & $63.57 \pm 10.74$ & $59.80 \pm 11.01$ \\
Sex (M/F) & 4 & 0 & 4 & 0.736 \\
Number of risk factors & $8 / 13$ & $34 / 15$ & $15 / 13$ & $37 / 15$ \\
Diabetes & 7 & 16 & 10 & 14 \\
Number of stenotic vessels & $1.76 \pm 0.70$ & $1.91 \pm 0.81$ & $1.75 \pm 0.75$ & $2.01 \pm 0.82$ \\
Number of stented vessels & $1.19 \pm 0.40$ & $1.30 \pm 0.46$ & $1.21 \pm 0.41$ & $1.21 \pm 0.41$ \\
Stents over 25 mm length & 16 & 2120 & 21 & 0.198 \\
Stents under 2.5 mm diameter & 0 & 2 & 3 & 0.344 \\
Multiple stents in one vessel & 2 & 8 & 6 & 0.787 \\
\hline
\end{tabular}




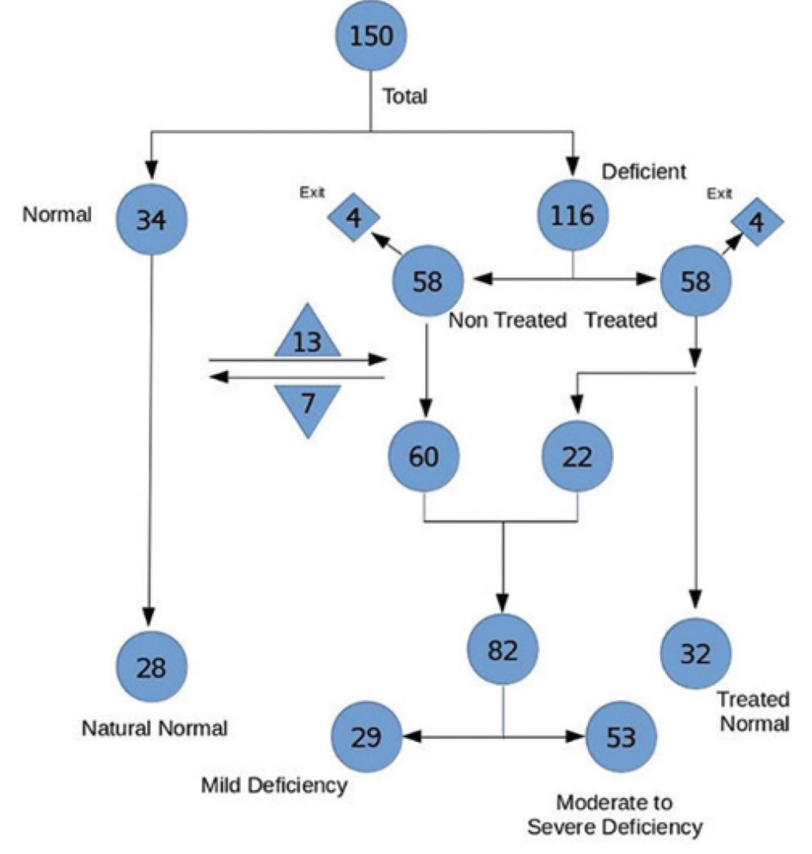

Figure 1. Flowchart of the Patients Included in the Study

a limited number of studies have been conducted on the relationship between vitamin D and restenosis. Yet, most studies were observational and have demonstrated reduced risk of MI, cardiac death, and restenosis. On the other hand, no Randomized Clinical Trials (RCTs) were found in this area in the literature (1-6). Only one animal study (on swine) was conducted whose results were in favor of our findings (6). The mentioned study showed that treatment of vitamin D deficiency could reduce restenosis of PCI.

The findings of our study indicated that Vitamin D supplementation made an improvement in only $43 \%$ of the patients. However, it seems that the used protocol was not enough for treating vitamin D deficiency in these patients. Thus, the protocol should be changed or more efficient protocols should be developed. In fact, the protocol should be revised according to baseline vitamin D classification and more severe deficient patients should receive higher dosages.

Our study results demonstrated that 7 out of the 142 patients had restenosis and 4 developed MACE including 2 expired subjects. None of the expired patients had normal vitamin $\mathrm{D}$ levels initially and none had final normal vitamin D levels using natural sunlight. Only one patient treated with vitamin D supplements had MACE. All the patients with MACE or restenosis had initial vitamin D deficiency. These results suggested that normal vitamin $\mathrm{D}$ was a protective factor against restenosis and MACE. The study findings also indicated that 28 patients finally reached normal vitamin $\mathrm{D}$ levels naturally with no MACE or restenosis. In contrast, 32 patients were deficient and reached normal levels by supplement, with one case of restenosis. The relationship between coronary artery stenosis and vitamin $\mathrm{D}$ has been proven for years. For instance, the Korean Longitudinal Study on Health and Aging demonstrated that vitamin D inadequacy was associated with significant coronary artery stenosis in an elderly community (15).

Although restenosis is the main complication of PCI, no
RCTs or human studies have been performed on this issue. Inherited factors may explain part of the risk of restenosis. Recently, vitamin D and its receptor (VDR) have been shown to be involved in modulating immune responses and cell proliferation, which is the main cause of restenosis. Monraats et al. (16) also demonstrated that vitamin D played an important role in restenosis after PCI. Therefore, vitamin $\mathrm{D}$ level and receptor genotype may be used as risk markers for restenosis and may contribute to individual patient screening prior to PCI in clinical practice. Gupta et al. (17) also demonstrated that significant decrease in vitamin $\mathrm{D}$ level and its receptor could initiate proliferating smooth muscle cells in neointimal lesions and increase atherogenic cytokines, such as TNF- $\alpha$. Thus, vitamin D deficiency potentiates development of coronary restenosis. They also studied other hormones related to calcium balance, such as calcitonin and parathormone, and suspected an underlying mechanism for uncontrolled growth of neointimal cells in injured arteries leading to restenosis (17).

This study had some limitations. In this study, stress echocardiography was used to diagnose restenosis and the patients underwent angiography if the result was positive. However, positive result in stress echocardiography does not exclude restenosis. Furthermore, carrying out the invasive diagnostic methods without having a good reason was not ethical and could impose various side effects and even cause death. Hence, future researches with longer follow-up periods must be conducted on the issue.

\subsection{Conclusion}

The results of this study showed that treatment of vitamin D deficiency could reduce restenosis of PCI. However, this protocol was only efficient for patients with moderate to severe deficiency and should be changed. Moreover, further detailed observations and trials with higher samples sizes are recommended to be performed on the issue.

\section{Acknowledgements}

There is no acknowledgement.

\section{Authors' Contribution}

Both authors contributed equally to this manuscript in all stages, including study concept and design, acquisition of data, analysis and interpretation of data, drafting of the manuscript, critical revision of the manuscript for important intellectual content, statistical analysis, administrative, technical, and material support, and study supervision.

\section{Financial disclosure}

There is no financial disclosure.

\section{Funding/Support}

This study was financially supported by Atherosclerosis Research Center, Baqiyatallah University of Medical Sciences, Tehran, Iran.

\section{References}

1. Holick MF. Vitamin D deficiency. NEngl J Med. 2007;357(3):266-81.

2. Holick MF, Chen TC. Vitamin D deficiency: a worldwide problem with health consequences. Am J Clin Nutr. 2008;87(4):1080S-6S.

3. Moradzadeh K, Larijani B, Keshtkar A, Hossein-Nezhad A, Rajabian 
R, Nabipour I, et al. Normative values of vitamin D among Iranian population: a population based study. International Journal of Osteoporosis \& Metabolic Disorders. 2008;1(1):8-15.

4. Bolland MJ, Grey A, Gamble GD, Reid IR. The effect of vitamin D supplementation on skeletal, vascular, or cancer outcomes: a trial sequential meta-analysis. Lancet Diabetes Endocrinol. 2014;2(4):307-20.

5. Byers T. Anticancer vitamins du Jour--The ABCED's so far. Am J Epidemiol. 2010;172(1):1-3.

6. Jozani Z, Assar O, Lotfian I. The effect of vitamin D deficiency on coronary artery stenosis severity in angioplasty patients in Baqiatallah hospital in 2013. Arak Medical University Journal. 2014;16(10):0-.

7. $\mathrm{Ku}$ YC, Liu ME, Ku CS, Liu TY, Lin SL. Relationship between vitamin D deficiency and cardiovascular disease. World J Cardiol. 2013;5(9):337-46.

8. Jorde R, Grimnes G. Vitamin D and metabolic health with special reference to the effect of vitamin D on serum lipids. Prog Lipid Res. 2011;50(4):303-12.

9. Artaza JN, Mehrotra R, Norris KC. Vitamin D and the cardiovascular system. Clin J Am Soc Nephrol. 2009;4(9):1515-22.

10. Jukema JW, Verschuren JJ, Ahmed TA, Quax PH. Restenosis after PCI. Part 1: pathophysiology and risk factors. Nat Rev Cardiol. 2012;9(1):53-62.
11. Razzouk L, Aggarwal S, Gorgani F, Babaev A. In-stent restenosis in the superficial femoral artery. Ann Vasc Surg. 2013;27(4):510-24.

12. Adams JS, Hewison M. Update in vitamin D. J Clin Endocrinol Metab. 2010;95(2):471-8.

13. Holick MF. Vitamin D and health: evolution, biologic functions, and recommended dietary intakes for vitamin D. Vitamin D: Springer; 2010. pp. 3-33.

14. Sicari R, Nihoyannopoulos P, Evangelista A, Kasprzak J, Lancellotti P, Poldermans D, et al. Stress Echocardiography Expert Consensus Statement--Executive Summary: European Association of Echocardiography (EAE) (a registered branch of the ESC). Eur Heart J. 2009;30(3):278-89.

15. Lim S, Shin H, Kim MJ, Ahn HY, Kang SM, Yoon JW, et al. Vitamin $\mathrm{D}$ inadequacy is associated with significant coronary artery stenosis in a community-based elderly cohort: the Korean Longitudinal Study on Health and Aging. J Clin Endocrinol Metab. 2012;97(1):169-78.

16. Monraats PS, Fang Y, Pons D, Pires NM, Pols HA, Zwinderman AH, et al. Vitamin D receptor: a new risk marker for clinical restenosis after percutaneous coronary intervention. Expert Opin Ther Targets. 2010;14(3):243-51.

17. Gupta GK, Agrawal T, Del Core MG, Hunter WJ, 3rd, Agrawal DK. Decreased expression of vitamin D receptors in neointimal lesions following coronary artery angioplasty in atherosclerotic swine. PLoS One. 2012;7(8):e42789. 